\title{
Temperature-induced tuning of emission spectra of liquid-crystal optical microcavities
}

\author{
Pavel Zemánek ${ }^{\mathrm{a}}$, Zdeněk Pilát ${ }^{\mathrm{a}}$, Jan Ježek ${ }^{\mathrm{a}}$, Silvie Bernatováa ${ }^{\mathrm{a}}$, Mehdi Aas ${ }^{\mathrm{b}}$, Alper Kiraz ${ }^{\mathrm{b}}$, and \\ Alexandr Jonášc \\ anstitute of Scientific Instruments of the CAS, v.v.i., Královopolská 147, 61264 Brno, Czech \\ Republic \\ ${ }^{\mathrm{b}}$ Department of Physics, Koç University, Rumelifeneri Yolu, Sariyer, 34450 Istanbul, Turkey \\ ${ }^{\mathrm{c}}$ Department of Physics, Istanbul Technical University, Maslak, 34469 Istanbul, Turkey
}

\begin{abstract}
Emulsion droplets of liquid crystals (LC) suspended in water and labeled with a suitable fluorescent dye can serve as active optofluidic microcavities, since the contrast of refractive index between the LC droplets and the surrounding aqueous medium allows excitation of whispering gallery modes (WGMs) in the droplets. In addition, such emulsion droplets can be also stably trapped in three-dimensions using optical tweezers which stabilizes the droplets while investigating their spectral characteristics. We explore various combinations of fluorescently dyed LC droplets and host liquid - surfactant systems and show that the WGM emission spectrum of an optically trapped LC droplet-based cavity can be largely and (almost) reversibly tuned by controlled changes of the ambient temperature that induce phase transitions in the LC droplets. Our results indicate feasibility of this approach for creating miniature tunable sources of coherent light.
\end{abstract}

Keywords: Optical microcavities, whispering gallery modes, optical trapping, liquid crystals, phase transition

\section{INTRODUCTION}

Optofluidics - a rapidly developing scientific discipline that combines the knowledge and experimental techniques of the physics of fluids, chemistry, and optics - utilizes optical and mechanical properties of fluids for creating unique reconfigurable optical systems inconceivable with conventional solid-state materials. ${ }^{1,2}$ Optical resonators (cavities) are among the most critical components of integrated optofluidic systems, as they are indispensable for implementing wavelength-selective optical devices such as add-drop filters, attenuators, or switches. ${ }^{3}$ Active fluid-based optical resonators containing a suitable gain medium then serve as the starting point for developing integrated optofluidic sources of laser light. ${ }^{4-7}$ Liquid microdroplets with nearly perfectly spherical geometry and atomically smooth surfaces, surrounded by a medium with a low refractive index, are capable of hosting high-quality optical resonances - whispering gallery modes (WGMs) - which arise from total internal reflection of light from the inner surface of the droplet confining a significant portion of the light intensity to the proximity of the surface. ${ }^{8,9}$ Thus, such microdroplets represent attractive self-assembled optofluidic cavities providing large flexibility in adjusting their optical properties, shape, and/or size which in turn allows for straightforward tuning of the cavity resonant spectrum. ${ }^{10-14}$

Liquid crystals (LCs) are complex fluids combining long-range organization found in crystalline solids with liquid-like fluidity that opens up the possibility of controlling the molecular orientation. In general, organization of LC molecules in any given state is characterized by the orientation of the molecular director field. ${ }^{15}$ Dielectric anisotropy of individual LC molecules in connection with their bulk and/or surface organization manifest themselves through optical birefringence of the LC fluid that can be adjusted by controlling orientation of LC molecules using external stimulus such as electric field, mechanical stress, or temperature changes. Consequently,

Further author information:

P.Z.: E-mail: zemanek@isibrno.cz

A.K.: E-mail: akiraz@ku.edu.tr

A.J.: E-mail: ajonas@itu.edu.tr

20th Slovak-Czech-Polish Optical Conference on Wave and Quantum Aspects of Contemporary Optics, edited by Jarmila Müllerová, Dagmar Senderáková, Libor Ladányi, L'ubomír Scholtz, Proc. of SPIE Vol. 10142, 101420F · @ 2016 SPIE · CCC code: 0277-786X/16/\$18 · doi: 10.1117/12.2262495

Proc. of SPIE Vol. 10142 101420F-1 
anisotropic optofluidic laser resonators formed by LC droplets offer additional paths for tuning the spectral profile of lasing emission. ${ }^{16,17}$

In this article, we report on the development and characterization of tunable optofluidic resonators based on dye-doped LC emulsion droplets suspended in an immiscible host liquid with the refractive index smaller than the refractive index of the LC material, thus supporting WGM resonances. The contrast of refractive index necessary for excitation of WGMs in the droplets also enables stable spatial confinement of the studied droplets in optical tweezers which adds large flexibility to the spectroscopic experiments. We explore various combinations of fluorescently dyed LC droplets and host liquid - surfactant systems and show that WGM emission spectrum of an LC droplet-based resonator can be largely and (almost) reversibly tuned by controlled changes of the ambient temperature that eventually induce phase transitions in the LC droplets. Our results indicate feasibility of this approach for creating miniature tunable sources of coherent light.

\section{EXPERIMENTAL SETUP}

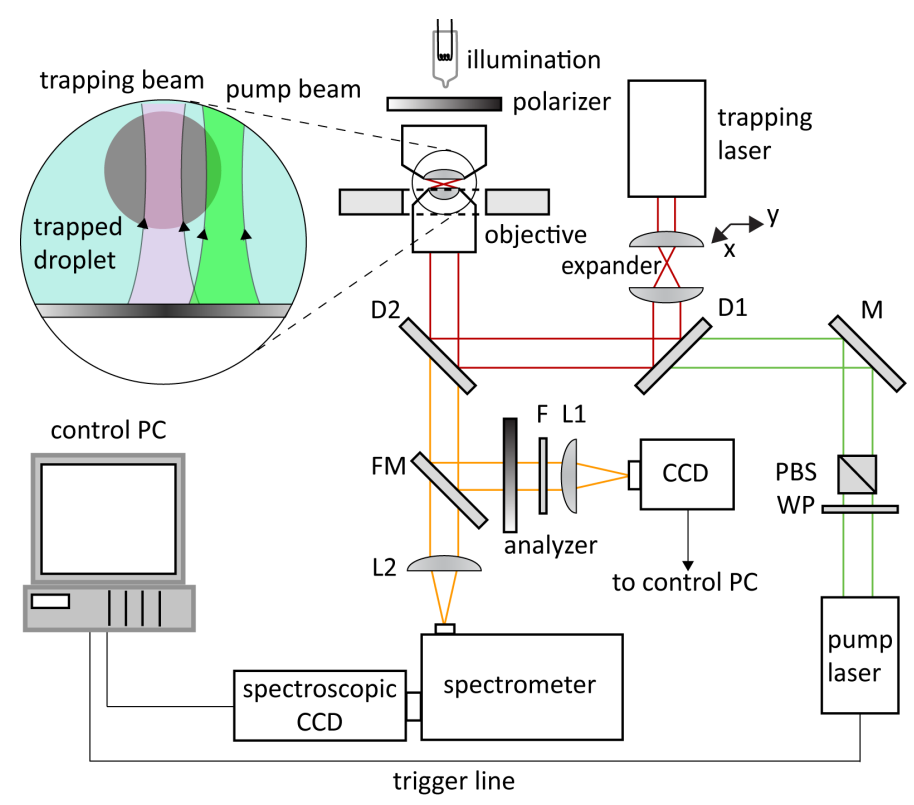

Figure 1. Experimental setup for optical spectroscopy of emulsion droplets of liquid crystals. The inset shows the detail of an optically trapped droplet with the pump laser beam positioned on the droplet rim. D1, D2: dichroic mirrors, F: band-pass filter, FM: flippable mirror, L1, L2: lenses, M: mirror, PBS: polarizing beam splitter, and WP: lambda-half wave plate.

Experimental setup used for the WGM spectroscopy of optically trapped emulsion droplets of liquid crystals is shown in Fig. 1. During spectral recordings, studied droplets were spatially confined using a single-beam optical tweezers (optical trap) generated by focusing an infrared cw laser beam (wavelength $1064 \mathrm{~nm}$, typical trapping power less than $500 \mathrm{~mW}$ at the sample) with a high numerical aperture oil-immersion microscope objective (magnification 100x, NA = 1.4). The trapping beam was expanded with a two-lens telescope to overfill the back aperture of the objective lens. In addition, transversal motion of the first lens of the telescope could be used for positioning of the optical trap in the field of view. ${ }^{18}$ In order to excite fluorescence in the optically trapped LC droplets, the droplets were pumped by a pulsed green laser beam (wavelength $532 \mathrm{~nm}$, pulse duration $9 \mathrm{~ns}$ ) focused near the droplet rim. A single pump pulse was sufficient to acquire an emission spectrum. The intensity of WGM emission from the droplets could be optimized by moving the optical trap with respect to the excitation beam focus. Fluorescence light emitted from the excited droplets was collected using the same microscope objective which was used for focusing the trapping and pump beams and subsequently analyzed by an imaging spectrometer (focal length $300 \mathrm{~mm}$ ). Simultaneously with the spectral acquisition, studied LC droplets could be observed using an inverted optical microscope equipped with a polarizer and analyzer with 
adjustable rotation angle. This enabled implementation of polarization microscopy which is especially well suited for examining the actual liquid-crystalline phase of the droplets during the experiment. Individual components of the setup were synchronized using a custom written LabView code.
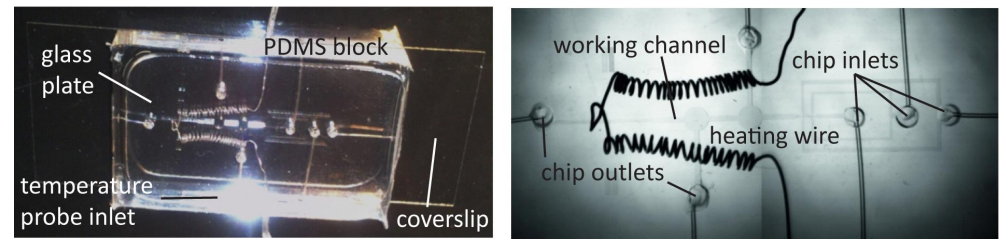

Figure 2. Microfluidic chip used for controlled heating of liquid crystal emulsions during spectroscopic experiments.

Figure 2 shows the prototype of a PDMS microfluidic chip which allowed in situ control of temperature of the studied liquid crystal emulsion. To this end, the chip featured a resistive-wire coil (heating wire) embedded in the PDMS block forming the body of the chip. This resistive wire coil was connected to an external DC power supply and - by controlling the power supply voltage - the amount of heat generated in the wire could be adjusted. The actual temperature of the chip could be monitored by a platinum resistance probe PT100 inserted into a dedicated inlet in the chip. Studied LC emulsions were loaded into the working channel in the chip (width $\sim 200 \mu \mathrm{m}$, height $\sim 50 \mu \mathrm{m}$ ) which was formed between the PDMS block and a standard microscope coverslip. This coverslip enabled optical access to the channel using inverted optical microscope configuration and it was irreversibly bonded to the bottom surface of the PDMS block. In order to prevent distortions of the chip during heating which stem from different heat expansion coefficients of PDMS and glass coverslip, a thick glass plate was embedded in the PDMS block to increase its bending rigidity. During a typical heating experiment, the studied droplet was confined by optical tweezers inside the working channel (see inset in Fig. 1). Occasional streaming of liquid in the channel could generate hydrodynamic forces that could pull the droplet out of the trap, thus disturbing the experiment. To increase the stability of the trapped droplet, thin flexible tubing was connected to both the inlet and the outlet of the chip. By manipulating the free ends of both pieces of tubing, hydrostatic pressure difference across the channel could be kept close to zero, thus eliminating the bulk fluid flow.

\section{EMULSION SYSTEMS OF LIQUID CRYSTALS}

Finding suitable combinations of liquid crystal, fluorescent dye, surfactant, and host liquid which allow generation of stable LC emulsion droplets in a well-defined liquid-crystalline phase is a necessary prerequisite for optical experiments involving study of emission from LC droplet-based optofluidic microcavities. Constraints on the used materials are mainly:

- Immiscibility of the used liquid crystal with the host liquid which determines the stability of the droplet size during the experiment

- Sufficient contrast of refractive indices of the liquid crystal $n_{\mathrm{LC}}$ and the host liquid $n_{\text {host }}$ to support resonant WGMs in the emulsion droplets $\left(n_{\mathrm{LC}}>n_{\text {host }}\right)$

- Good solubility of the used dye in the liquid crystal

To address these issues, we have prepared and characterized the following emulsions of dye-doped liquid crystals in an aqueous host liquid $\left(n_{\text {host }}=1.33\right)$ :

a) $5 \mathrm{CB}$ liquid crystal (refractive index in isotropic phase $n_{\mathrm{LC}}=1.58$ at $640 \mathrm{~nm}$ ) $+1 \% \mathrm{w} / \mathrm{w}$ Nile Red fluorescent dye $+4 \mathrm{mM}$ sodium dodecyl sulfate (SDS) surfactant. At room temperature around $\sim 23^{\circ} \mathrm{C}$, this material combination produces LC emulsion droplets with radial orientation of the molecular director (see a typical droplet image in the inset of Fig. 3(a)) 
(a)

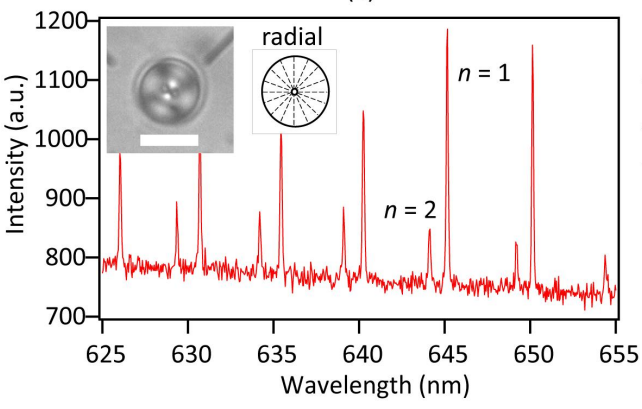

(c)

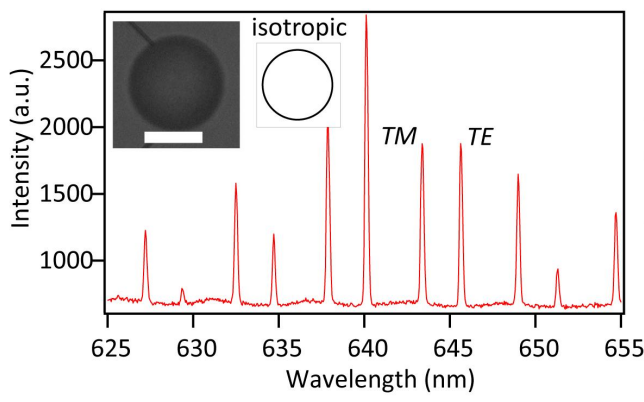

(e)

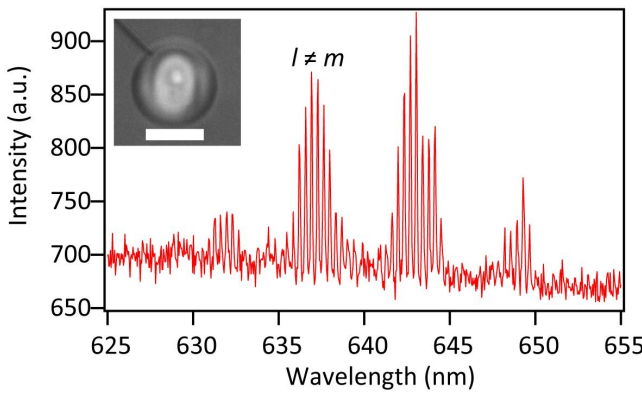

(b)

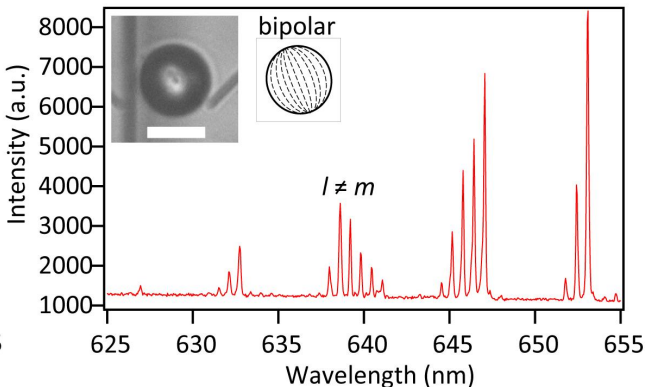

(d)

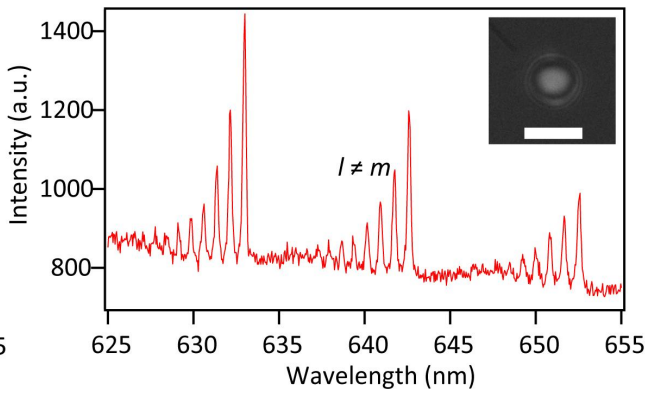

(f)

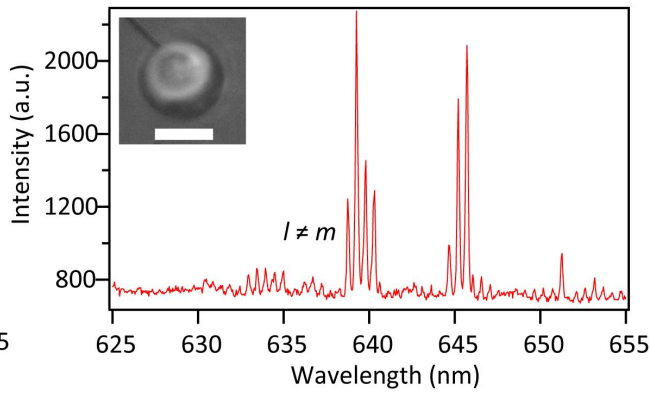

Figure 3. Emission spectra recorded from various dye-doped emulsion droplets of liquid crystals pumped with a pulsed laser beam. (a) 5CB + Nile Red $+4 \mathrm{mM}$ SDS; (b) 5CB + Nile Red + $0.7 \mathrm{mM}$ SDS; (c) $5 \mathrm{CB}+\mathrm{Nile}$ Red $+>1200 \mathrm{mg} / \mathrm{L}$ Triton X100; (d) 5CB + Nile Red + 300 mg/L Triton X100; (e) PCBN + Nile Red + $1200 \mathrm{mg} / \mathrm{L}$ Triton X100; (f) 7CB + Nile Red $+1200 \mathrm{mg} / \mathrm{L}$ Triton X100. Insets show typical droplet images acquired for the given emulsion system using polarization microscopy and schematics of spatial distribution of the LC molecular director. Scale bars: $10 \mu \mathrm{m}$.

b) $5 \mathrm{CB}+1 \% \mathrm{w} / \mathrm{w}$ Nile Red $+0.7 \mathrm{mM}$ SDS. At room temperature around $\sim 23{ }^{\circ} \mathrm{C}$, this material combination produces LC emulsion droplets with bipolar orientation of the molecular director (see a typical droplet image in the inset of Fig. 3(b)).

c) $5 \mathrm{CB}+1 \% \mathrm{w} / \mathrm{w}$ Nile Red $+>1200 \mathrm{mg} / \mathrm{L}$ Triton X100 surfactant. At room temperature above $\sim 23^{\circ} \mathrm{C}$, this material combination produces LC emulsion droplets in isotropic (melted) phase (see a typical droplet image in the inset of Fig. 3(c)).

d) $5 \mathrm{CB}+1 \% \mathrm{w} / \mathrm{w}$ Nile Red $+300 \mathrm{mg} / \mathrm{L}$ Triton X100. At room temperature around $\sim 23^{\circ} \mathrm{C}$, this material combination produces LC emulsion droplets with bipolar orientation of the molecular director (see a typical droplet image in the inset of Fig. 3(d)).

e) PCBN liquid crystal (refractive index in isotropic phase $n_{\mathrm{LC}}=1.58$ at $640 \mathrm{~nm}$ ) $+1 \% \mathrm{w} / \mathrm{w}$ Nile Red + $1200 \mathrm{mg} / \mathrm{L}$ Triton X100. At room temperature around $\sim 23{ }^{\circ} \mathrm{C}$, this material combination produces LC 
emulsion droplets with bipolar orientation of the molecular director (see a typical droplet image in the inset of Fig. 3(e)).

f) $7 \mathrm{CB}$ liquid crystal (refractive index in isotropic phase $n_{\mathrm{LC}}=1.56$ at $\left.640 \mathrm{~nm}\right)+1 \% \mathrm{w} / \mathrm{w}$ Nile Red + $1200 \mathrm{mg} / \mathrm{L}$ Triton X100. At room temperature around $\sim 23^{\circ} \mathrm{C}$, this material combination produces LC emulsion droplets with bipolar orientation of the molecular director (see a typical droplet image in the inset of Fig. 3(f)).

Droplet emission spectra shown in Fig. 3 reflect significant differences in the internal long-range order of liquid crystal molecules within the droplets in individual studied cases. With radial orientation of LC molecular director (Fig. 3(a)), LC droplets display complete spherical symmetry. Thus, all WGMs with the same angular mode number $l$, which is approximately equal to the number of light wavelengths that fit into the resonant light path around the droplet circumference, are frequency-degenerate. Radial orientation of LC director is associated with strong birefringence which causes a large difference in the ordinary refractive index $n_{o}$ sensed by the $T E-$ polarized modes (electric field tangential to the droplet surface) and the extraordinary refractive index $n_{e}$ sensed by the $T M$-polarized modes (electric field normal to the droplet surface). Since $n_{o}<n_{e}$, in small radial LC droplets with diameters $\sim 10 \mu \mathrm{m}$, the contrast of the effective refractive indices between the droplet and the host liquid (refractive index $n_{\text {host }}$ ) is sufficient to support the $T M$-polarized modes but not the $T E$-polarized modes which, therefore, are virtually absent from the spectrum. As the droplet size grows, quality factor ( $Q$-factor) of the droplet WGM resonances generally increases and the $T E$-polarized modes are eventually expected to appear in the WGM spectrum; however, their amplitude is always smaller than the amplitude of the corresponding $T M$-polarized modes due to the unfavorable contrast of refractive index. ${ }^{16}$ In addition to $T M$-polarized WGMs with the lowest radial mode number $n=1$ which circulate closest to the droplet surface, radial LC droplets can also support higher-order radial modes with $n=2$; these higher-order radial modes typically display lower $Q$-factors than $n=1$ modes. $^{16}$

For bipolar orientation of LC director (Fig. 3(b), (d), (e), (f)), spherical symmetry of the resonator is lost and modes with the same angular mode number $l$, but different azimuthal mode numbers $m$, experience different profiles of refractive index during their propagation around the droplet. Thus, frequency degeneracy of WGMs observed in Fig. 3(a) is lifted and modes with $l \neq m$ appear at different wavelengths. ${ }^{19,20}$ This degeneracy lifting manifests itself as splitting of single spectral peaks into peak families whose precise structure including mode spacing and relative intensities depends on the actual internal profile of the refractive index within the droplet.

Finally, for isotropic-phase droplets (Fig. 3(c)), long-range order of LC molecules is completely lost due to Brownian motion at elevated temperature, and the LC droplets essentially behave like droplets of regular liquids. In particular, birefringence disappears and both $T M$-polarized and $T E$-polarized modes with comparable intensities can be now supported. ${ }^{8}$ Absence of long-range order also restores complete spherical symmetry of the droplet and, thus, the WGMs become frequency-degenerate.

Because of the reproducibility of preparation and overall stability, we chose to work with the radial-phase emulsions of LC droplets prepared using the recipe described in item a) of the above list of various LC droplet emulsions.

\section{TEMPERATURE-INDUCED TUNING OF EMISSION SPECTRA OF LC DROPLETS}

Using temperature-controlled microfluidic chip described in Sec. 2, we characterized the changes of WGM emission spectra of LC droplets accompanying phase transitions of the droplets from liquid-crystalline phase (temperature below the LC clearing point) into the isotropic phase (temperature above the LC clearing point) and back during repeated heating and cooling of the chip containing the studied LC emulsion. To this end, 5CB liquid crystal with $1 \% \mathrm{w} / \mathrm{w}$ Nile Red dye was emulsified in $4 \mathrm{mM}$ SDS/water host liquid. As described in Sec. 3, this combination of materials produces LC droplets in the radial liquid crystalline phase at the ambient room temperature around $\sim 23^{\circ} \mathrm{C}$.

Figure 4 presents typical fluorescence emission spectra recorded from an optically trapped LC droplet of diameter approximately $13.5 \mu \mathrm{m}$ at different stages of the heating/cooling cycle, with the actual chip temperature 
(a) $\mathrm{T}=37.9^{\circ} \mathrm{C}$ (radial phase)

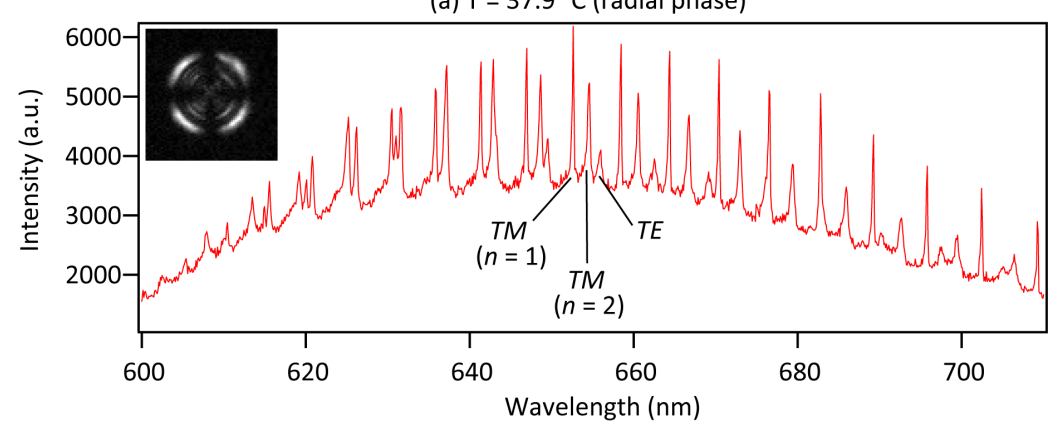

(b) $\mathrm{T}=40.9^{\circ} \mathrm{C}$ (isotropic phase)

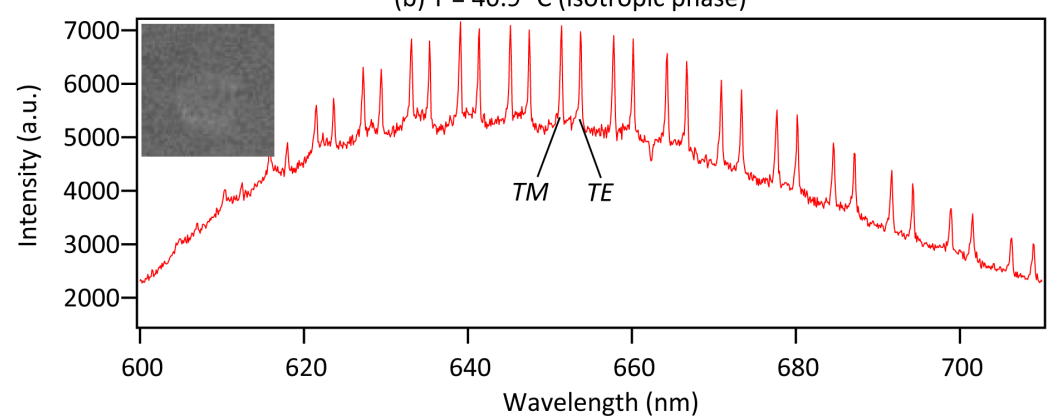

(c) $\mathrm{T}=39.9^{\circ} \mathrm{C}$ (radial phase)

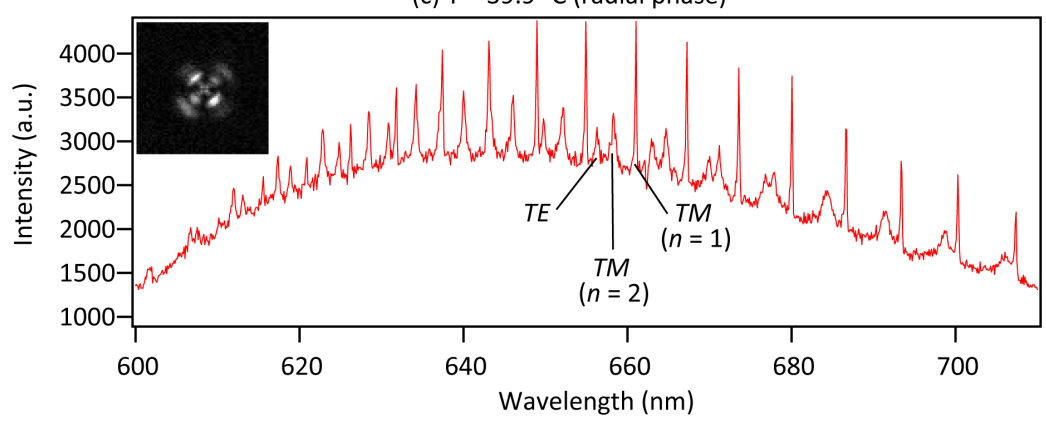

Figure 4. Tuning of fluorescence emission spectrum of LC droplet by controlled temperature changes. For explanation, see discussion in the text.

indicated in the plot headings. Inset in each spectrum shows the corresponding image of the studied droplet acquired using polarization microscopy which reports on the internal organization of LC molecules within the droplet. In the radial LC phase where the molecular director is highly organized, pointing radially away from the center of the droplet, the image of the droplet is characterized by a symmetric cross-shaped appearance. At the same time, the droplet emission spectrum features $T M$-polarized modes of radial orders $n=1,2$ and also $T E$-polarized modes of the lowest radial order $n=1$ (Fig. 4(a)). Due to the low contrast between the ordinary refractive index of liquid crystal molecules $n_{o}$ sensed by the $T E$-modes and refractive index of the surrounding host medium $n_{\text {host }}$, the intensities and $Q$-factors of the $T E$-modes are lower than those observed for the $T M$-modes (see discussion in Sec. 3 for details). Upon melting and transition into the isotropic phase which occurs when the clearing temperature of $\sim 40.1^{\circ} \mathrm{C}$ is reached, droplet birefringence resulting from the long-range order of LC molecules vanishes and the droplet observed through crossed polarizer and analyzer becomes practically invisible (Fig. 4(b)). At the same time, $T M$-polarized modes with $n=2$ disappear and the intensities and $Q$-factors of $T E$-polarized modes with radial order $n=1$ become comparable to those of the corresponding $T M$-modes. This behavior stems from the fact that both $T E$ - and $T M$-mode families now sense the same average refractive index $n_{\mathrm{LC}}$ of the isotropic phase $\mathrm{LC}$, where $n_{o}<n_{\mathrm{LC}}<n_{e}$. When the temperature is lowered and the droplet transitions back to the liquid crystalline phase, its internal structure becomes visible 
again and its spectrum returns to the original WGM pattern with high intensity and high $Q$-factor $T M$-polarized modes of various radial orders and low intensity and low $Q$-factor $T E$-polarized modes of the lowest radial order (Fig. 4(c)).

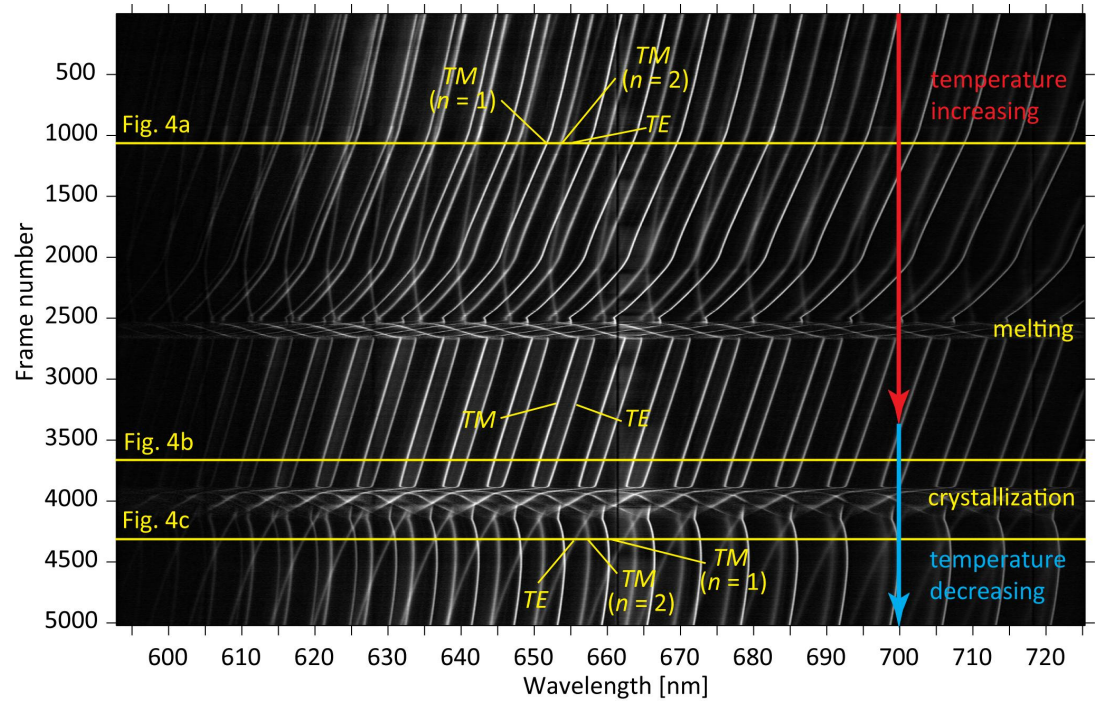

Figure 5. Complete tuning cycle of WGM emission spectrum of an LC droplet induced by controlled temperature changes. Yellow horizontal lines correspond to the LC droplet emission spectra shown in Fig. 4 . Labels indicate typical $T M-$ and $T E$-polarized modes which display different tuning trends during temperature changes.

The complete temperature-tuning cycle, example spectra from which are shown in Fig. 4, is summarized in the two-dimensional spectral plot of Fig. 5. In this temperature-tuning cycle, emulsion temperature first gradually increases from $\sim 37.5^{\circ} \mathrm{C}$ to $\sim 41.1^{\circ} \mathrm{C}$ (frames $1-3400$ ), crossing the $\mathrm{LC}$ clearing point of $\sim 40.1^{\circ} \mathrm{C}$ at the frame $\sim 2500$. After reaching the peak value at the frame $\sim 3400$, the temperature starts decreasing, crosses the LC crystallization point at the frame $\sim 3900$, and reaches the final value of $\sim 39.9^{\circ} \mathrm{C}$ at the frame 5022 . During the whole experiment, all modes display a variable-rate drift to the blue end of the spectrum which can be attributed to the gradual droplet dissolution in the host liquid. ${ }^{20}$ Besides this dissolution-related effect, a closer look at the two-dimensional spectral plot reveals that $T M$ - and $T E$-polarized modes are being tuned in opposite directions; with increasing temperature, the wavelength of $T M$-modes decreases whereas the wavelength of $T E$ modes increases and with decreasing temperature, the tuning directions switch. This trend can be explained by different dependence of the ordinary and extraordinary refractive indices $n_{o}, n_{e}$ on temperature. When the temperature rises, long-range organization of LC molecules gradually disappears and the ordinary refractive index $n_{o}$ starts increasing, approaching the value of the isotropic-phase refractive index $n_{\mathrm{LC}}$. Thus, WGMs of the given angular mode number $l$ corresponding to $T E$-polarized resonances that sense the refractive index $n_{o}$ are shifted to longer wavelengths. Conversely, gradual loss of LC order causes decrease of the extraordinary refractive index $n_{e}$ towards the isotropic-phase value of $n_{\mathrm{LC}}$; hence, $T M$-polarized resonances, whose spectral positions are dictated by the value of $n_{e}$, shift to shorter wavelengths. Naturally, the trends reverse when the temperature decreases.

A video file illustrating the progress of the full WGM tuning cycle accompanying reversible phase transition of an optically trapped LC droplet from radial LC phase to isotropic phase and back can be accessed through the link provided in Fig. 6. This video displays simultaneously the actual droplet WGM spectrum, temperature of the emulsion, and corresponding polarization image of the droplet acquired during various phases of the melting/crystallization process. As shown in the video, upon crossing the phase transition points (frames $\sim 2500$ and $\sim 3900$ ), the dynamics of spectral changes becomes very fast, with WGMs rapidly shifting in different directions. These large spectral shifts correlate directly with major variations in the internal structure of the trapped LC droplet revealed by polarization microscopy. Thus, spectroscopic measurements can potentially serve as a sensitive indicator of phase transition processes taking place in LC droplets. 


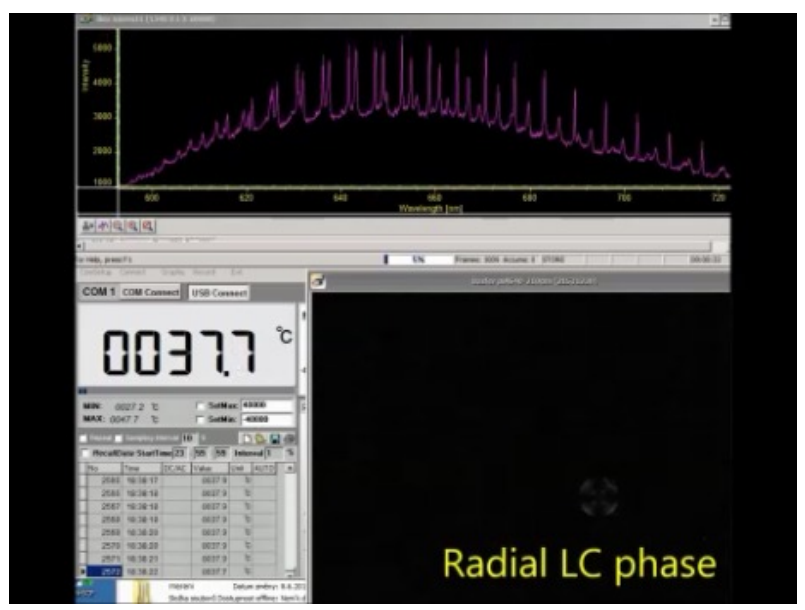

Figure 6. "Video 1" Complete tuning cycle of emission spectrum of an LC droplet induced by controlled temperature changes. Droplet spectra are shown together with the corresponding temperatures of the emulsion and polarization images of the studied droplet. http://dx.doi.org/10.1117/12.2262495.1

\section{CONCLUSION}

We have studied tunable optofluidic microcavities formed by dye-doped emulsion droplets of liquid crystals suspended in an aqueous host liquid and confined in optical tweezers. We have shown that WGM emission spectra of such droplets can be monitored over extended time periods, having recorded several thousands of spectra from a single droplet. By changing the temperature of the emulsion, we have shown that the WGM spectra of the droplet-based resonators can be largely and almost reversibly tuned in both directions. Residual irreversibility observed in the spectral tuning patterns can be potentially reduced or even eliminated by optimizing the composition of the host liquid supporting the droplets (in particular, the type and/or concentration of the used surfactant). The presented results indicate feasibility of this approach for creating miniature largely tunable sources of coherent light. In addition, spectroscopic measurements with LC droplets subject to external stimuli (e.g. controlled changes of the ambient temperature that eventually induce phase transitions in the droplets) can elucidate the physical processes taking place in the droplets, thus contributing to the fundamental research in the field of liquid crystal physics.

\section{ACKNOWLEDGMENTS}

We acknowledge financial support from Türkiye Bilimsel ve Teknolojik Araştirma Kurumu (TÜBİTAK) (114F253), COST Action (MP1205), the Ministry of Education, Youth and Sports of the Czech Republic (LD14069, LO1212), and the European Commission (CZ.1.05/2.1.00/01.0017).

\section{REFERENCES}

[1] Monat, C., Domachuk, P., and Eggleton, B. J., "Integrated optofluidics: A new river of light," Nat. Photonics 1, $106-114$ (2007).

[2] Fan, X. and White, I. M., "Optofluidic microsystems for chemical and biological analysis," Nat. Photonics 5, 591-597 (2011).

[3] Vahala, K. J., "Optical microcavities," Nature 424, 839 (2003).

[4] Azzouz, H., Alkhafadiji, L., Balslev, S., Johansson, J., Mortensen, N. A., Nilsson, S., and Kristensen, A., "Levitated droplet dye laser," Opt. Express 14, 4374-4379 (2006).

[5] Kiraz, A., Sennaroglu, A., Doganay, S., Dundar, M. A., Kurt, A., Kalaycioglu, H., and Demirel, A. L., "Lasing from single, stationary, dye-doped glycerol/water microdroplets located on a superhydrophobic surface," Opt. Commun. 276, 145-148 (2007). 
[6] Nizamoglu, S., Gather, M. C., and Yun, S. H., "All-biomaterial laser using vitamin and biopolymers," Advanced Materials 25, 5943-5947 (2013).

[7] Jonáš, A., Aas, M., Karadă̆, Y., Manioğlu, S., Anand, S., McGloin, D., Bayraktar, H., and Kiraz, A., "In vitro and in vivo biolasing of fluorescent proteins suspended in liquid microdroplet cavities," Lab on a Chip 14, 3093 - 3100 (2014).

[8] Righini, G. C., Dumeige, Y., Feron, P., Ferrari, M., Conti, G. N., Ristic, D., and Soria, S., "Whispering gallery mode microresonators: Fundamentals and applications," Rivista Del Nuovo Cimento 34, 435-488 (2011).

[9] Qian, S.-X., Snow, J. B., Tzeng, H. M., and Chang, R. K., "Lasing droplets: Highlighting the liquid-air interface by laser emission," Science 231, 486 (1986).

[10] Kiraz, A., Kurt, A., Dündar, M. A., and Demirel, A. L., "Simple largely tunable optical microcavity," Appl. Phys. Lett. 89, 081118 (2006).

[11] Kiraz, A., Karadă̆, Y., and Coskun, A. F., "Spectral tuning of liquid microdroplets standing on a superhydrophobic surface using electrowetting," Appl. Phys. Lett. 92, 191104 (2008).

[12] Kiraz, A., Karadag, Y., Yorulmaz, S. C., and Muradoglu, M., "Reversible photothermal tuning of a salty water microdroplet," Phys. Chem. Chem. Phys. 11, 2597-2560 (2009).

[13] Yorulmaz, S. C., Mestre, M., Muradoglu, M., Alaca, B. E., and Kiraz, A., "Controlled observation of nondegenerate cavity modes in a microdroplet on a superhydrophobic surface," Opt. Commun. 282, 30243027 (2009).

[14] Aas, M., Jonáš, A., Kiraz, A., Brzobohatý, O., Ježek, J., Pilát, Z., and Zemánek, P., "Spectral tuning of lasing emission from optofluidic droplet microlasers using optical stretching," Opt. Express 21(18), 2138021394 (2013).

[15] Muševič, I., "Nematic colloids, topology, and photonics," Phil. Trans. R. Soc. A 371, 20120266 (2013).

[16] Humar, M., Ravnik, M., Pajk, S., and Muševič, I., "Electrically tunable liquid crystal optical microresonators," Nat. Photonics 3, 595-600 (2009).

[17] Humar, M. and Muševič, I., "Surfactant sensing based on whispering-gallery-mode lasing in liquid-crystal microdroplets," Opt. Express 19, 19836-19844 (2011).

[18] Aas, M., Jonáš, A., and Kiraz, A., "Lasing in optically manipulated, dye-doped emulsion microdroplets," Opt. Commun. 290, 183-187 (2013).

[19] Hossein-Zadeh, M. and Vahala, K. J., "Fiber-taper coupling to whispering-gallery modes of fluidic resonators embedded in a liquid medium," Opt. Express 14, 10800-10810 (2006).

[20] Jonáš, A., Karadag, Y., Mestre, M., and Kiraz, A., "Probing of ultrahigh optical Q-factors of individual liquid microdroplets on superhydrophobic surfaces using tapered optical fiber waveguides," J. Opt. Soc. Am. B 29, 3240-3247 (2012). 